\title{
Theoretical-Experimental Analyses of Simple Geometry Saturated Conductivities for a Newtonian Fluid
}

\author{
Mário Augusto Camargo, Paulo Cesar Facin, ${ }^{*}$ and Luiz Fernando Pires \\ Department of Physics, State University of Ponta Grossa (UEPG), 84.030-900, Ponta Grossa, PR, Brazil
}

(Received on 1 December, 2009)

\begin{abstract}
The conductivity $(K)$ of porous media represents an important physical parameter in several areas of knowledge. In saturated flow, the saturated conductivity $\left(K_{0}\right)$ is the most important parameter of porous system and it is related to the fluid and porous media properties. In order to evaluate the potential of a new tool for measuring $K_{0}$, such as the computational simulation with Boltzmann models for fluid flows, two experiments were carried out using two simplified media: 1) a cylindrical cavity and 2) a cavity having a parallelepiped shape. Both have simple geometries that allow analytical $K_{0}$ solutions in order to compare with the experimental and simulated results. Glycerin was used as infiltrate fluid due to its high viscosity that permits laminar flows and the use of Darcy's law to evaluate $K_{0}$. The results demonstrate a good agreement among techniques (experimental, computational, and analytical) of $K_{0}$ determination for cavities that present Reynolds number $(\mathrm{Re})$ smaller than one.
\end{abstract}

Keywords: Boltzmann model, Darcy's law, porous media.

\section{INTRODUCTION}

Conductivity $(K)$ represents a physical parameter which defines the easiness with which a fluid can flow throw a certain porous media and it depends on the media properties as well as on the percolating fluid [1]. Factors related to the porous media structure such as types of porous, pore size distribution, number of pores, tortuosity and connectivity directly affect the $K$ values [2,3]. Conductivity achieves its maximum value when the porous media is saturated $\left(K_{0}\right)$ and its value decreases quickly as moisture decreases.

Conductivity direct measurements usually demand a lot of work, are expensive and time consuming. For this reason some authors have proposed theoretical models to estimate this physical property. For porous media such as soil, Alexander \& Skaggs [4] presented a theoretical model for the hydraulic conductivity measurement of non saturated soils from the retention curve data considering that water flows through capillary tubes. Falleiros et al. [5] analyzed the spatial and temporal variability of $K$ in relation to the water distribution in the soil using an exponential model. Zhuang et al. [6] developed a theoretical model to estimate $K$ in non saturated soil from some of its parameters and compared their results with those obtained by other models.

Another alternative to measure $K$ in porous media is the one that uses computational simulation. There are a lot of works which use simulations such as the Boltzmann model to obtain $K$ from porous media. Some works use pore structures obtained through computerized tomography [7]. Other works use structures reconstructed from porous and connectivity information obtained from 2D images [8].

The Boltzmann model used to determine $K$ computationally is able to simulate dynamically the mass transport as well as the momentum, which in this case is the NavierStokes equation (NS equation). The model is based on the evolution of a relaxation equation for a fluid particle distribution in a discrete spatial lattice, in which relaxation time defines viscosity.
The main objective of this work was to evaluate the Boltzmann model potential to obtain saturated conductivity of empty cavities such as cylinders and boxes. This objective was achieved through comparisons among $K_{0}$ (experimental, analytical, and simulated) values of simple geometries when a Newtonian fluid flows through them.

\section{MATERIAL AND METHODS}

\subsection{Experimental Setup}

The device used for the $K_{0}$ experimental measurement basically consists of a Mariotte tube and a 'drainage hole' system (Figure 1). The height of the 'drainage hole' imposes a constant $H$ hydraulic load on the investigated empty cavities (Figure 2). This system can be coupled either to the cylinder or to the box. Experiments were carried out with glycerin with three different viscosity values.

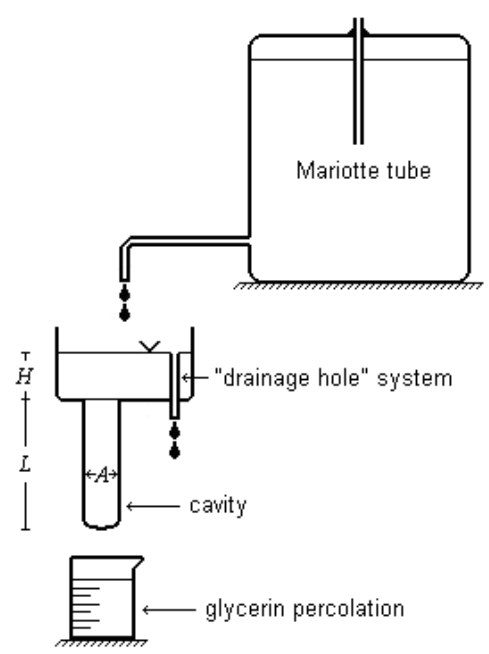

FIG. 1: Schematic drawn of the experimental setup used in the $K_{0}$ (saturated conductivity) experimental measurements. 
The choice of the glycerin is because the Darcy's law is not universally valid for all conditions of liquid flow in porous media. As the cavities are empties if water was used as percolating fluid the flow velocity it would be very high. In this specific case the linearity of the flux versus hydraulic gradient relationship fails. In the experiment the Reynolds number $(R e)$ was determined to guarantee the use of the Darcy's law to evaluate $K_{0}(\operatorname{Re}<1)$.

The cylinder and box have the following dimensions: $0.45 \mathrm{~cm} \times 20.5 \mathrm{~cm}$ (diameter $\mathrm{x}$ length) and $0.635 \mathrm{~cm} \times$ $0.635 \mathrm{~cm} \times 4.7 \mathrm{~cm}$ (width $\times$ height $\times$ length).

The experimental procedure involved the following steps: 1) saturation of the empty cavities by glycerin; 2) glycerin viscosity measurement by the Stokes method; 3) glycerin viscosity measurement by the cylinder method [9]; 4) measurement of glycerin percolated through cavities to obtain its volume; 5) measurement of time interval for glycerin percolation; and 6) use of the Darcy's law to calculate the saturated conductivity $K_{0}\left(\mathrm{~cm} \mathrm{~s}^{-1}\right)$ of the empty cavity:

$$
K_{0}=\frac{V}{A t} \frac{L}{(L+H)}
$$

where $V\left(\mathrm{~cm}^{3}\right)$ is the percolated glycerin volume, $A\left(\mathrm{~cm}^{2}\right)$ is the cross section area, $t(s)$ the percolated volume time interval, $L(\mathrm{~cm})$ is the height and $H(\mathrm{~cm})$ is the glycerin column height above the cavity (Figure 1).



(a)

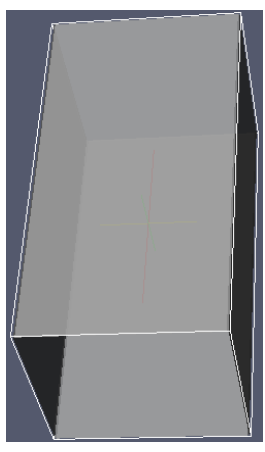

(b)
FIG. 2: Empty cavities used for the evaluation of $K_{0}$ (saturated conductivity). (a) cylinder and (b) box.

The measurements of cavities $K_{0}$ and the glycerin kinematic viscosity $v\left(\mathrm{~cm}^{2} \mathrm{~s}^{-1}\right)$ occurred at the same temperature, which was monitored with the $0.1{ }^{\circ} \mathrm{C}$ resolution.

\subsection{Analytical solutions}

The cylinder and box $K_{0}$ is obtained by solving the NS equation for a Newtonian fluid in an incompressible regime and a non-slip condition on the walls $[10,11]$. The $K_{0}$ analytical expression for a $d$ diameter cylinder is well known and is presented in equation (2):

$$
K_{0 c y l i n d e r}=\frac{d^{2}}{32} \frac{g}{v}
$$

in which $g\left(\mathrm{~cm} \mathrm{~s}^{-2}\right)$ is the gravity acceleration.

For a box with a side square straight section area $l(\mathrm{~cm})$ the analytical expression for $K_{0}$ is given by [10]:

$$
K_{0 b o x}=\frac{l^{2}}{12} \frac{g}{v}\left(1-6 \sum_{n=1}^{\infty} \frac{\tanh \alpha_{n}}{\alpha_{n}^{5}}\right),
$$

in which $\alpha_{n}=(2 n-1) \frac{\pi}{2}, \quad n=1,2, \ldots$

\subsection{Numerical simulation}

For $K_{0}$ values obtained through computational methods, the Boltzmann model was used. The three-dimensional physical space represented in the simulation, necessarily has to be discretized and a three-dimensional lattice is used for this. The sites in this lattice might represent solid and pores. Being a site in the lattice located by the vector $\vec{X}$ and having $b_{m}$ neighbors, the evolution equation for the particle distribution function $N_{i}(\vec{X}, T)$ is given by the so-called "Lattice Boltzmann Equation":

$$
N_{i}\left(\vec{X}+\vec{c}_{i}, T+1\right)=N_{i}(\vec{X}, T)+\Omega_{i}(\vec{X}, T),
$$

where $T$ is the time discrete variable, $i$ is the direction of one of the closest $b_{m}$ neighbors, $\vec{c}_{i}$ the velocity vector in direction $i$, with $i=0$ representing the $b_{r}$ resting particles.

The dynamics occurs in two steps in this model, the collision and the propagation steps. Collision is the step that simulates the molecular collisions needed so that thermodynamic equilibrium occurs; this step is represented by the term $\Omega_{i}(\vec{X}, T)$ in equation (4), called collision operator. At the collision step time is not incremented. This step is local and does not involve interactions with neighboring sites:

$$
N_{i}^{\prime}(\vec{X}, T)=N_{i}(\vec{X}, T)+\Omega_{i}(\vec{X}, T),
$$

The variable $N_{i}^{\prime}(\vec{X}, T)$ is called "collided" distribution function and represents a new value at the same site $\vec{X}$ and the same step of time $T$.

Equation (4) represents the propagation step, it transports the collided term $N_{i}^{\prime}(\vec{X}, T)$ to the neighboring site $\vec{X}+\vec{c}_{i}$, at posterior time $T+1$. A simple and sufficient form of collision operator which recovers the NS macroscopic equation is known as $\mathrm{BGK}^{1}$ [12] operator:

$$
\Omega_{i}=\frac{N_{i}^{e q}-N_{i}}{\tau}
$$

where $\tau$ is the relaxation time, which regulates the number of time steps that the $N_{i}$ particle distribution takes to get closer to the equilibrium distribution $N_{i}^{e q}$. That is, if $N_{i}<N_{i}^{e q}, \Omega_{i}>$ 0 and the term $\Omega_{i}$ will be added to $N_{i}$ making $N_{i}$ tend to $N_{i}^{e q}$, the same analysis might be done for the case in which $N_{i}>N_{i}^{e q}$.

\footnotetext{
${ }^{1}$ Varibles $\vec{X}$ and $T$ were omitted to reduce notations.
} 
The particle density $\rho$ in a site and the momentum, in this method are given by:

$$
\begin{gathered}
\sum_{i=0}^{b} N_{i}=\rho, \\
\sum_{i=1}^{b_{m}} N_{i} \vec{c}_{i}=\rho \vec{u} .
\end{gathered}
$$

Taking that into consideration and imposing conservation of mass and momentum to the collision operator:

$$
\begin{gathered}
\sum_{i=0}^{b} \Omega_{i}=0 \\
\sum_{i=1}^{b_{m}} \Omega_{i} \vec{c}_{i}=0
\end{gathered}
$$

where $b=b_{r}+b_{m}$.

Particle distribution for the $N_{i}^{e q}$ is usually obtained through the $N_{i}^{e q}$ expansion, in power series at the macroscopic velocity $\vec{u}$, being $O\left(u^{2}\right)$ sufficient so that NS equation is recovered. In this case, demands on $N_{i}^{e q}$, besides conditions given by equations (7) and (8) are that the pressure be independent from macroscopic velocity and obey the flow density tensor of the momentum:

$$
\sum_{i=0}^{b_{m}} N_{i}^{e q} c_{i \alpha} c_{i \beta}=p \delta_{\alpha \beta}+\rho u_{\alpha} u_{\beta}
$$

where $c_{i \alpha}$ is the $\alpha$ component of $c_{i}$ and the hydrostatic pressure $p$ is given by:

$$
p=\frac{b_{m} c^{2}}{b D} \rho
$$

where $D$ is the Euclidian dimension of space in which the lattice is immerse and $c^{2}=\left|c_{i}\right|^{2}$. With this, the balance distribution form for moving particles is given by:

$N_{i}^{e q}=\frac{\rho}{b}+\frac{\rho D}{b_{m} c^{2}} c_{i \alpha} u_{\alpha}+\frac{\rho D(D+2)}{2 b_{m} c^{4}} c_{i \alpha} u_{\alpha} c_{i \beta} u_{\beta}-\frac{\rho D}{2 b_{m} c^{2}} u^{2}$.

In the main directions $x, y$ and $z$ the equilibrium distribution must be doubled $\left(N_{i}^{e q}=2 N_{i}^{e q}\right)$ so that viscosity is isotropic [13].

Resting particles have the following equilibrium distribution:

$$
N_{o}^{e q}=\frac{\rho}{b} b_{r}-\frac{\rho}{c^{2}} u^{2}
$$

For a macroscopic analysis of the dynamics proposed by equation (4) time $\delta$ and space scales $h$ are usually used and the Knudsen variable $k_{n}=\frac{h}{L}=\frac{\delta}{T_{c}}$ is defined, where $L$ and $T_{c}$ are, respectively, the macroscopic characteristic length and time. With this, the Chapman-Enskog [14] method can be used, considering the equilibrium distribution disturbance, to show that equation (4) becomes the Navier-Stokes, given by equation (15), disregarding the $\mathrm{O}\left(k_{n}^{2}\right)$ contributions:

$$
\partial_{t}\left(\rho u_{\beta}\right)+\partial_{\alpha}(p) \delta_{\alpha \beta}+\partial_{\alpha}\left(\rho u_{\alpha} u_{\beta}\right)=v \rho \partial_{\alpha}\left(\partial_{\alpha} u_{\beta}+\partial_{\beta} u_{\alpha}\right),
$$

in which $\alpha$ and $\beta$ are indexes that represent the spatial coordinates $x, y$ or $z$. For these indexes Einstein's notation is seen (repeated indexes sum). The equation (15) is the $\beta$ component of the NS equation with kinematic viscosity $v$ given by:

$$
v=\frac{h^{2} c^{2}}{\delta(D+2)}\left[\tau-\frac{1}{2}\right]
$$

The boundary conditions used in the simulation are periodic, that is, the fluid which leaves one end of the cavity is injected in the other end. The interaction between fluid and solid occurs so that there is no sliding, in this case, the 'bounce back' condition was adopted, in which a fluid particle that collides with the walls has its velocity inverted. The program return permeability $k$, which is calculated (equation 17) with the Santos et al. [8] method, where in a stationary flow, the momentum applied to the fluid is equal to the loss of momentum on the walls:

$$
k=v \phi \frac{\left\langle m u_{x}\right\rangle}{\left\langle m u_{x}\right\rangle_{\text {lost }}},
$$

in which $\phi$ is the porosity, $\left\langle m u_{x}\right\rangle$ is the fluid average momentum in the cavity and $\left\langle m u_{x}\right\rangle_{\text {lost }}$ is the average momentum lost by the fluid in the collisions with the cavity walls in a propagation step.

In order to obtain $K$ from the permeability the relation below is used:

$$
K=k \frac{g}{v}
$$

\section{RESULTS AND DISCUSSION}

The experimental saturated conductivity values $\left(K_{0 \exp }\right)$, analytical $\left(K_{\text {Oanal }}\right)$ and simulated $\left(K_{0 \text { simul }}\right)$ for both empty cavities, as well as the simulated permeability $\left(k_{\text {simul }}\right)$, are presented in table 1 .

The $K_{\text {Oanal }}$ value for the cylinder is found through equation (2), assuming $d=0.45 \mathrm{~cm}$. The determination of $K_{0 \text { anal }}$ for the box is carried out through equation (3), considering $l=$ $0.635 \mathrm{~cm}$, where the two first terms in the infinite series are sufficient to obtain good approximation.

In simulations the necessity to represent cavities (Figure 2) through a discrete lattice, raises the need of evaluating a minimum value for the cavities dimensions in which the discretization problems are minimized. The value of 200 sites for the lateral dimension $l$ of the box was chosen based on results of figure 3 , where the permeability dependence $k$ is shown, calculated in the simulation for a square box, according to the side $l$ of the box (for the cylinder, the same behavior is observed).

The scale factor used in simulations was $h=\frac{0.635}{200}=$ 0.003175 for the box and $h=\frac{0.45}{200}=0.00225$ for the cylinder.

The velocity fields for cavities of different sizes are shown in figure 4, these fields are not accessible in most experiments. 
TABLE 1: Saturated conductivity $\left(K_{0}\right)$ results for the three analyzed methods (analytical, experimental, and simulated). The value of gravity acceleration used in the calculations was $979 \mathrm{~cm} . \mathrm{s}^{-2}$

\begin{tabular}{ccccccc}
\hline & $\begin{array}{c}\mathrm{K}_{0_{\text {exp }}} \\
\left(\mathrm{cm} \mathrm{s}^{-1}\right)\end{array}$ & $\begin{array}{c}\mathrm{K}_{0_{\text {anal }}} \\
\left(\mathrm{cm} \mathrm{s}^{-1}\right)\end{array}$ & $\begin{array}{c}\mathrm{K}_{0_{\text {simul }}} \\
\left(\mathrm{cm} \mathrm{s}^{-1}\right)\end{array}$ & $R e_{\text {exp }}$ & $\begin{array}{c}k_{\text {simul }} \\
\left(\mathrm{cm}^{2}\right)\end{array}$ & $\begin{array}{c}v \\
\left(\mathrm{~cm}^{2} \mathrm{~s}^{-1}\right)\end{array}$ \\
\hline Cylinder & $0.8 \pm 0.06$ & 0.8 & $0.8 \pm 0.04$ & 0.2 & 0.0064044 & $8.3 \pm 0.3$ \\
Box 1 & $1.5 \pm 0.08$ & 1.5 & $1.5 \pm 0.01$ & 0.2 & 0.0141724 & $9.5 \pm 0.1$ \\
& & & & & & \\
Box 2 & $6.7 \pm 0.08$ & 4.2 & $4.2 \pm 0.09$ & 1.6 & 0.0141724 & $3.3 \pm 0.3$ \\
\hline
\end{tabular}

Errors of $K_{0}$ (experimental and simulated) and $v$ were obtained by error propagation.



FIG. 3: Simulated permeability $(k)$ values in function of the box width (1).
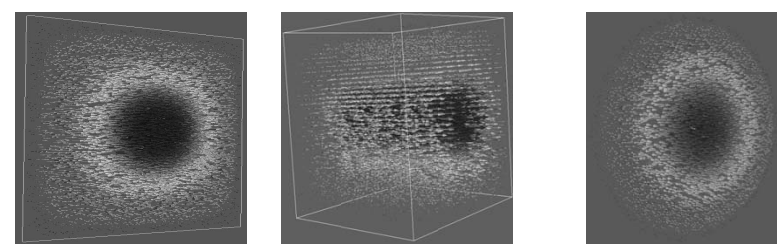

FIG. 4: Velocity fields for the cavities with different dimensions. Greater and darker arrows represent higher velocities. (a) Box with dimensions of 200 sites $\times 200$ sites $\times 1$ site; (b) and $40 \times 40 \times 40$ and (c) cylinder with 200 diameter and 1 high.

A constant control of temperature was kept in the experiments aiming at minimizing the variations in the $K_{0}$ measurement, since viscosity, which originated from molecules collision, suffers great variations with the temperature and strongly influences the $K_{0}$ results.

The Boltzmann model simulates the NS equation because its dynamics respects the mass conservation principles as well as the momentum, being such conditions fundamental to the Newtonian fluid flow at a constant temperature. Therefore, simulations captured the physics relevant to the flow in the experimental arrangement of the tested cavities, presenting excellent agreement with experimental results, validating the model.

Once flows of interest in the soil physics area are framed in the flow profile addressed by this work, water flow in soil samples usually occur with $\operatorname{Re}<1$ [15]. As $K$ experimental measurements usually present difficulties, numeric simulation might be an interesting alternative for future predictions of this physical property without the need to collect samples and experimental measurements. This way, Boltzmann method appears as an alternative tool which might contribute with the development of this knowledge area in the future.

\section{CONCLUSIONS}

Results of this work lead to the following conclusions:

1. Fluid viscosity monitoring during $K_{0}$ experimental measurements compensates possible errors due to temperature variations;

2. Results obtained show good agreement of the three methods of $K_{0}$ measurement (analytical, experimental, and simulated) when $\operatorname{Re}<1$. For box 2, whose $\operatorname{Re}>1$, percentage deviation between simulated (or analytical) and experimental was $59.5 \%$;

3. Boltzmann model is a tool with potential for further work involving $K_{0}$ measurements for more complex porous media.

\section{Acknowledgements}

To Brazilian National Counsel of Technological and Scientific Development (CNPq) for the PQ grant, to Araucária 
Foundation and Capes for the master scholarship.

To Drs. Luiz Emerich dos Santos and Fabiano Gilberto Wolf of the Laboratory of Porous Media and Thermophysics
Properties (LMPT) of UFSC (Federal University of Santa Catarina) by the theoretical contributions.
[1] A. Klute, C. Dirksen, Hydraulic conductivity and diffusivity: Laboratory methods. In: BLACK, C.A. (Ed.) Methods of Soil Analysis. I. Physical and Mineralogical Methods. Madison, ASA/SSSA, 1986. pp.697-734.

[2] K. Reichardt, L.C. Timm, Solo, planta e atmosfera: conceitos, processos e aplicações. Barueri: Manole, 2004. 478p.

[3] F.A.M. Cássaro, L.F. Pires, R.A. Santos, D. Gimenez, K. Reichardt, Funil de Haines modificado: curvas de retenção de solos próximos à saturação. Revista Brasileira de Ciência do Solo, 32:2555-2562, 2008.

[4] L. Alexander, R.W. Skaggs, Predicting unsaturated hydraulic conductivity from the water characteristic. Transactions of the ASAE, 29:176-184, 1986.

[5] M.C. Falleiros, O. Portezan, J.C.M. Oliveira, O.O.S. Bacchi, K. Reichardt, Spatial and temporal variability of soil hydraulic conductivity in relation to soil water distribution, using an exponential model. Soil Technology, 45:279-285, 1998.

[6] J. Zhuang, K. Nakayama, G.R. Yu, T. Miyazaki, Predicting unsaturated hydraulic conductivity of soil based on some basic soil properties. Soil and Tillage Research, 59:143-154, 2001.

[7] M.-E. Kutay, A.H. Aydilek, E. Masad, Laboratory validation of lattice Boltzmann method for modeling pore-scale flow in granular materials. Computers and Geotechnics, 33:381-395, 2006.
[8] L.O.E. Santos, C.E.P. Ortiz, H.C. Gaspari, G.E. Haverroth, P.C. Philippi, Prediction Of Intrinsic Permeabilities With Lattice Boltzmann Method In: 18TH International Congress of Mechanical Engineering, 2005, Ouro Preto. Proceedings of COBEM 2005. Rio de Janeiro: ABCM, 2005. v. 1.

[9] P.L. Libardi, Dinâmica da água no solo. Piracicaba, 2004. P. L. Libardi, 2004. 420p.

[10] T.C. Papanastasiou, C.G. Georgios, N.A. Andreas, Viscous Fluid Flow. Boca Raton: CRC Press, 2000. 275 p.

[11] M.C. Potter, D.C. Wiggert, Mecânica dos Fluidos. São Paulo, Cengance Learning, 2009. 223p.

[12] Y.H. Qian, D. D'humieres, P. Lallemand, Lattice BGK Models for Navier-Stokes Equation. Europhysics Letters, 17:479-484, 1992.

[13] D.H. Rothman, S. Zaleski, Lattice-gas cellular automata: simple models of complex hydrodynamics. United Kingdom: Cambridge University Press, 1997. 297p.

[14] P.C. Facin, Modelo de Boltzmann Baseado em Mediadores de Campo Para Fluidos Imiscíveis. Tese de Doutorado, Programa de Pós-Graduao em Engenharia Mecânica, Universidade Federal de Santa Catarina, Florianópolis, 2003.

[15] J. Bear, Dynamics of fluids in porous media. New York, Dover1, 1988, 119p. 